\title{
触 New Disease Reports \\ First report of Golovinomyces sordidus causing powdery mildew on plantain in Vietnam
}

\author{
L.T.T. Tam ${ }^{1 *}$ and H.V. Cuong ${ }^{2}$ \\ ${ }^{1}$ Division of Plant Pathology, Plant Protection Research Institute (PPRI); Duc Thang, Bac Tu Liem, Hanoi, Vietnam; ${ }^{2}$ \\ Research Centre for Tropical Plant Pathology (RCTPP), Vietnam National University of Agriculture (VNUA), Trau Quy, Gia \\ Lam, Hanoi, Vietnam
}

*E-mail: thanhtamle10_2012@yahoo.co.uk

Received: 27 Aug 2015. Published: 20 Dec 2015. Keywords: Erysiphe sordida, medicinal crops, Plantago major

Plantago major (plantain) is one of the most abundant and widely distributed medicinal crops in the world. Since 2013, typical symptoms of powdery mildew were observed in plantain growing wild in fields as well as cultivated for medicine production, the latter with a large area in Hanoi, Vietnam. Disease incidence was observed at around $80 \%$ and the disease severity almost $100 \%$ with almost all aerial parts of the plant (i.e. flowers, leaves and stem), covered by white fungal colonies (Fig. 1). As a result, yield at harvest was dramatically decreased. A representative diseased specimen was deposited in the PPRI Herbarium (Accession No. PPRIPM050).

Conidiophores were composed of one to three cells, and measured (82.4-)112.2-147.1(-172.1) $\mu \mathrm{m}$ long. Footcells of conidiophores were cylindrical, straight, or curved at the base and 50-62.5 x 10-12.5 $\mu \mathrm{m}$ long (Fig. 2). Conidia, produced in chains, were variable in shape, oval to cylindrical, oval or doliform, 30-35 x 17.5-20 $\mu \mathrm{m}$ with a length/width ratio of 1.6 to 2.0. No chasmothecia were found. The morphological characteristics were consistent with descriptions of the genus Golovinomyces (Braun \& Cook, 2012). To confirm the identity of the causal fungus to the species level, the complete ITS region of rDNA from diseased plant accession PPRI-PM050 was amplified with our universal primer pairs HF1 (5'-GGATCCTCGTAACAAGGTTTCCGTAG-3')/ HR4 (5'-CTGCAGCTCCGCTTATTGATATGCTT-3') (Tam et al., 2015) and directly sequenced. The resulting sequence $(636 \mathrm{bp})$ was deposited in GenBank (Accession No. KM260734). A BLAST search using the present data revealed that the ITS sequence shares $99 \%$ identity with those of Erysiphe sordida and Golovinomyces sordidus (AF011309, AB077658 respectively) in which E. sordida and G. sordidus are synonyms (Saenz \& Taylor, 1999; Matsuda \& Takamatsu, 2003)

Pathogenicity was confirmed through inoculation by gently pressing

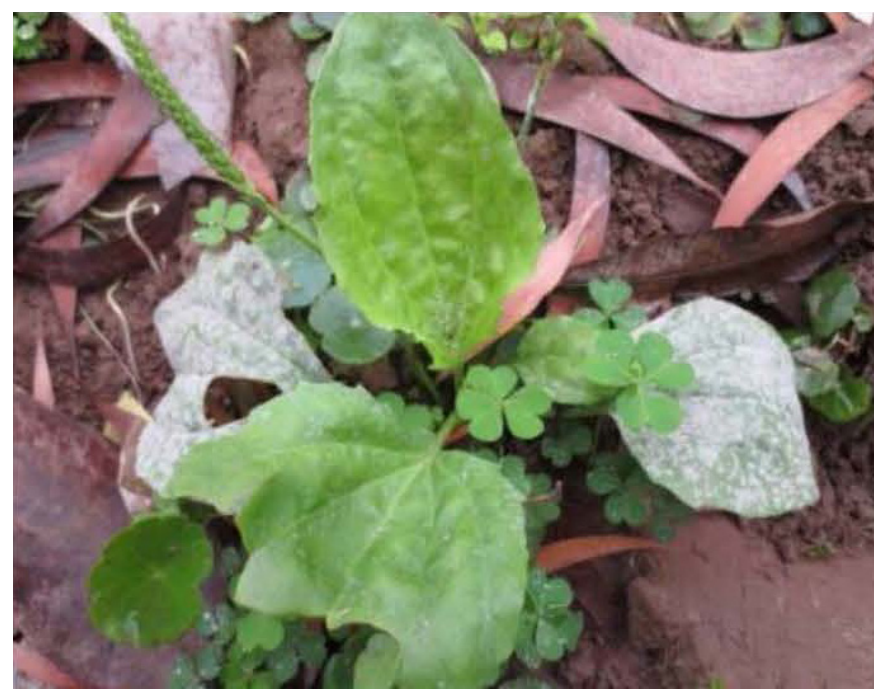

Figure 1 diseased leaves of plantain onto young leaves of ten potted three-week-old seedlings of $P$. major. Ten non-inoculated seedlings were used as controls. Plants were maintained in a greenhouse in PPRI at $26-28^{\circ} \mathrm{C}$. Inoculated leaves developed symptoms after nine days, whereas the control plants remained symptomless. The fungus present on the inoculated leaves was morphologically identical to that observed on the original diseased leaves, with the same sequence following PCR as above, fulfilling Koch's postulates. G. sordidus is also known to infect several host plants in the Plantaginaceae and is widely distributed in North America, Europe, Central Asia, China, Japan, Korea and New Zealand (Braun \& Cook, 2012). To our knowledge, this is the first report of G. sordidus infecting $P$. major in Vietnam. The disease seems to be an important threat to the production of pharmaceuticals from plantain in Vietnam.

\section{References}

Braun U, Cook RTA, 2012. Taxonomic Manual of

the Erysiphales (Powdery Mildews), CBS Biodiversity Series No. 11. Utrecht, The Netherlands: CBS-KNAW Fungal Biodiversity Centre.

Matsuda S, Takamatsu S, 2003. Evolution of host-parasite relationships of Golovinomyces (Ascomycete: Erysiphaceae) inferred from nuclear rDNA sequences. Molecular Phylogenetics and Evolution 27, 314-327.

http://dx.doi.org/10.1016/S1055-7903(02)00401-3

Saenz GS, Taylor JW, 1999. Phylogeny of the Erysiphales (powdery mildews) inferred from internal transcribed spacer ribosomal DNA sequences. Canadian Journal of Botany 77, 150-168. http://dx.doi.org/10.1139/b98-235

Tam LTT, Dung PN, Liem NV, 2015. First report of powdery mildew caused by Erysiphe cruciferarum on Brassica juncea in Vietnam. Plant Disease (in press). http://dx.doi.org/10.1094/PDIS-06-15-0678-PDN

To cite this report: Tam LTT, Cuong HV, 2015. First report of Golovinomyces sordidus causing powdery mildew on plantain in Vietnam. New Disease Reports 32, 33. http://dx.doi.org/10.5197/j.2044-0588.2015.032.033

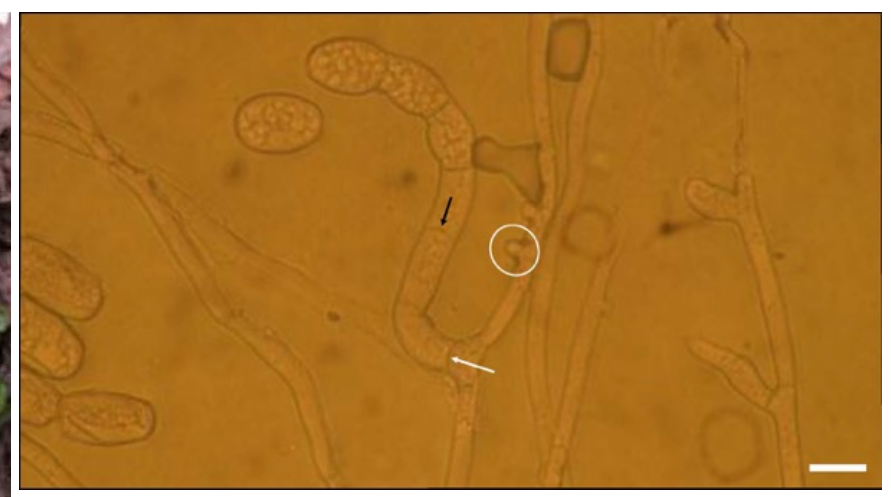

Figure 2 